\title{
Point of Care Testing and Transfusion Safety in Resource Limited Settings: A Review
}

\author{
Massimo La Raja ${ }^{1,2^{*}}$, Roberto Musi ${ }^{3}$, Mauro Fattorini ${ }^{1}$, Elisa Piva ${ }^{4}$ and Giovanni Putoto ${ }^{1}$ \\ ${ }^{1}$ Doctors with Africa, NGO CUAMM, Padova, Italy \\ ${ }^{2}$ Department of Immunohaematology and Transfusion Medicine, Arzignano, Italy \\ ${ }^{3}$ Italian Association of Clinical Engineers, ICCS Group, Clinical Engineers for the Cooperation and Development, Pavia, Italy \\ ${ }^{4}$ Department of Laboratory Medicine, Padova Hospital-University, School of Medicine, Padova, Italy
}

"Corresponding author: Massimo La Raja, Ospedale di Arzignano, via del Parco 1, 36071 Arzignano, (Vicenza) Italy, Tel: +0039(0)444-479310; Fax: +0039(0)444-479275; E-mail: massimo.laraja@ulss5.it

Received date: Feb 17, 2015, Accepted date: Apr 13, 2015, Publication date: Apr 16, 2015

Copyright: (C 2015 Raja ML, et al. This is an open-access article distributed under the terms of the Creative Commons Attribution License, which permits unrestricted use, distribution, and reproduction in any medium, provided the original author and source are credited.

\begin{abstract}
Worldwide, one of the fastest growing aspects of clinical laboratory testing is point of care testing (POCT). Decentralized patient care and access to testing in under-served areas are key elements in the evolving expansion of POCT. Several available POCT devices can potentially contribute to safer transfusion practices in resourcelimited settings. Only "rapid diagnostic tests" and some simple hemoglobinometers are normally encountered in the peripheral hospitals of low income countries. Strengths, weaknesses and barriers of large scale utilization of POCT in transfusion medicine are discussed.
\end{abstract}

Keywords: Transfusion safety; Hemoglobinometers; Anaemia; Developing countries

\section{Introduction}

There is a growing interest in new or rediscovered laboratory technologies that can be utilized without the presence of specialized personnel, at the bedside and/or in extreme field conditions, with limited or no infrastructure and without specialized maintenance services. Point of care testing (POCT) allows for rapid and accurate laboratory testing at the bedside where immediate and effective clinical decisions need to be made. This can be performed at community level, in operating rooms or in complex emergency settings. These diagnostic devices are increasingly recognized as appropriate in low and middle income countries, where human, financial and logistic recourses are scarce, and where there are major constraints in supply and maintenance services $[1,2]$. ASSURED the acronym that has been proposed to summarize the main criteria that define POCT devices stands for: affordable, sensitive, specific, user friendly, rapid and robust, equipment free and delivered [3]. The spectrum of available technologies varies from low-tech to high tech, and their effective introduction depends on the specific target product profiles [4], i.e. their intended settings. Examples of POCT devices that are increasingly utilized in high income countries include dry chemistry analyzers, rapid immunological tests, coagulometers and hematology analyzers. Other devices, like CD4 counters and Nucleic Acid Amplification Tests -NAAT-, are more specifically conceived for infectious diseases control programs in developing countries [5].

In the hospital setting blood transfusion is an essential treatment that is available worldwide; however the more current, sophisticated and high throughput technologies utilized in transfusion medicine in high income countries are hardly suitable for blood banks in resourceconstrained health services. In these contexts few blood units are collected and transfused daily and often only in emergencies, therefore instruments and devices that allow single or small batch testing and rapid turnaround time are required. Global improvement in transfusion safety depends on technologies that can be safely operated in disadvantaged situations.

\section{Study Design and Methods}

We examine the contribution of POCT in the four main areas of transfusion medicine: hematology, hemostasis, infectious diseases screening and pre-transfusion testing. We address strengths and weaknesses of POCT transfusion medicine, as well as factors that may influence their widespread implementation in resource-limited settings. PubMed was searched from January 1st, 1990, to February 28th 2014, with the terms "point-of-care" and "transfusion", "point-ofcare" and "hematology" and "rapid tests" and "transfusion" for all available articles. We selected reports, reviews and, epidemiological studies published in English and we also reviewed references from selected publications. The titles and abstracts of each article were screened for relevance with regard to the limited-resource setting, the identified articles were retrieved for assessment of the full text.

\section{Results}

507 articles were initially identified utilizing the search criteria. Further selection based on their relevance in the context of resourcelimited settings reduced the number to 84 papers which were reviewed and organized according to the four areas of interest in transfusion medicine.

\section{Hematology}

Rapid and reliable hemoglobin level testing is the cornerstone for appropriate blood transfusion and therefore represents the first step in the transfusion process. The Global Negleted Tropical Diseases (NTD) program launched the requirements for a method to measure 
hemoglobin concentrations that should be accurate enough to detect the anticipated changes in haemoglobin levels induced by interventions for NTDs, should not require mains electricity, can be performed with minimal training and supervision and uses whole blood so that no dilution steps are required [6].

There are several options of POCT in hematology some of which have been marketed for decades. A review was recently published [7] which details the options available for POCT in hematology. Essentially they are divided in single parameter, i.e. hemoglobinometers, and multi-parameters analyzers.

Traditional and more recent "visual" hemoglobinometers, such as Shali, Lovibond and the Hemoglobin Color Scale, are simple devices which are however too subjective and inaccurate to correctly identify severely anemic patients that may require $\mathrm{RBC}$ transfusion [8-10]. A new POC visual method that can also be interpreted via smartphone has been recently tested and has given some promising results [11].

More sophisticated and reliable point-of-care photometric hemoglobinometers have been utilized for decades for POCT in different settings. The haemoglobin method that has been most widely used in NTD programmes is the HemoCue system. The HemoCue system $^{\circ}$ (HemoCue, Angelholm, Sweden) provides a reliable, rapid one-step haemoglobin determination with a sensitivity of $80-96 \cdot 6 \%$ compared to standard laboratory methods $[12,13]$. The method utilizes dedicated microcuvettes suitable for direct hemoglobin determination from undiluted venous or capillary blood. The main advantages are that it uses battery power, is easy of use, accurate, provides rapid results, and is portable [14]. As well as being accurate the HemoCue method is robust and has an in-built quality checking tool [15]. New versions of these portable photometric analyzers, the HemoCue Hb 301 system and The DiaSpect Hemoglobin T system (DiaSpect Medical, GmBH, Sailauf, Germany) offer the additional advantage of utilizing "reagent free" microcuvettes that can be stored in high temperatures and in high humidity conditions, situations that are not uncommon in developing countries [16]. The main disadvantages of these portable hemoglobinometers are represented by their cost and scarce availability at the local level [17]. Another method, the Haemoglobin Colour Scale (HCS) has been designed for field situations in resource-poor countries and is considered to be better than clinical diagnosis in detecting anaemia in children and pregnant women $[18,19]$. The main disadvantages are the need for a specific type of chromatography paper as well as good natural light. The method is not able to detect incremental changes in haemoglobin less than $1 \mathrm{~g} / \mathrm{dl}$. The Lovibond comparator method is an alternative technique for measuring haemoglobin that does not require a dilution step, may have satisfactory precision and accuracy, but requires an exact volume of $0.03 \mathrm{ml}$ of whole blood, which is difficult to achieve in field situations [20]. The Microhematocrit method is another costeffective alternative for hemoglobin estimation. Although the method is sufficiently accurate and it utilizes fairly cheap consumables $[21,22]$ it requires a basic laboratory infrastructure and a reliable power supply. More recently a portable hemoglobinometer that utilizes reagent strips was marketed offering another technological alternative. The TrueHb Hemometer, developed by Ambar Srivastava of the Indian Institute of Technology (IIT), is a relatively small device and represents the first case of an innovation by the biomedical engineering department of IIT-Delhi [23].

Noninvasive devices offer an appealing alternative for haemoglobin determination, however limitations in accuracy, especially in emergency situations such as hypovolemic shock, caution against their utilization as unique transfusion decision tools [24-26].

In recent years simple, compact and affordable multiplatform hematology analyzers that utilize impedance cell counting methodology and spectrophotometric hemoglobin determination have become available [6]. "Impedence" based counters require a supply of dedicated and relatively bulky "liquid" kits as well as regular maintenance and a reliable power supply. All these characteristics make these instruments less appropriate and manageable in remote settings. The microhematocrit based automated "quantitative buffy coat"-QBC-method offers an alternative to traditional multiparameter hematology analyzers. The main advantages of this system are that it is portable and relies only on "dry" reagents. This analyzer still has a fairly limited distribution and utilization in resource-limited settings [27] notwithstanding its simplicity and remarkable performance.

Finally as far as hemoglobinometers are concerned it should be mentioned that a rapid assessment of hemoglobin concentration is usually performed before bleeding the donor. In this context the accuracy of the test result is less critical and many of the basic abovementioned methods, including the simple Hemoglobin Color Scale, can play a role in the selection of blood donors in resourcelimited settings $[28,29]$.

\section{Hemostasis}

Since blood components such as fresh frozen plasma and platelet concentrates are scarce in peripheral and resource limited settings, point-of-care devices and methods for coagulation and platelet function testing are only briefly presented in this review.

Simple bedside whole blood coagulation utilizing a dry tube is a basic and very simple method that was employed in the past to detect coagulopathy and is still utilized in cases of snake bites in Africa and elsewhere [30,31]. It has been demonstrated that a 20 minute whole blood clotting test -20WBCT- shows good correlation with fibrinogen concentration, however its clinical predictivity in the case of snake bites has been questioned [32].

In high income countries much more sophisticated devices for POC coagulation testing are available. Their main uses are the monitoring of vitamin-K antagonist oral anticoagulants, the assessment of platelet function, in particular in patients undergoing anti-platelets therapy, as well as the rapid evaluation of clotting function in bleeding patients during surgery and in emergency settings [33-37]. Out of these the viscoelastometric POCT devices are particularly useful in giving quick information on all the main phases and components of clot formation, including fibrinogen concentration, platelet activity and fibrinolysis [38]. For this reason they are utilized to guide the clinical management of acute coagulopathy that follows trauma and hemorrhages, including obstetric cases [39-41]. To our knowledge however there are no studies that document the utilization or the appropriateness of POC coagulation tests in resource-limited settings. As in the case of other more sophisticated POC devices cost and unavailability of supplies at local level are likely to represent major barriers to their utilization. As far as the management of coagulopaties is concerned, it is important to remember that in most peripheral hospitals in Sub-Saharan Africa whole blood is the only available blood product [42]. Fresh whole blood units, if available in sufficient number, are indeed an effective therapy in case of dilutional and consumption coagulopathy that follows severe acute bleeding and trauma since it rapidly restores 
simultaneously red blood cells, active clotting factors and platelets [43].

\section{Transfusion transmitted infections -“TTIs"- screening}

According to global standards all blood units must be tested at least for HIV antibodies - HIV, Ab- hepatitis B antigen S - Hb Ag-, Hepatitis C antibodies -HCV Ab- and Syphilis. Other infections, such as malaria and Chagas disease, can be screened according to the epidemiological context [44]. In high income countries immunoenzymatic or chemioluminescence tests are routinely employed for TTIs screening. These sophisticated and often automated laboratory platforms require constant maintenance, regular supply of dedicated reagents and controls and must be operated by skilled laboratory personnel. All these features make their utilization and reliable functioning difficult in resource-limited and/or remote settings.

Since the late eighties Rapid Diagnostic Tests - RDTs - have been available for HIV diagnosis, and have represented a breakthrough in transfusion safety in low income countries [45-49]. Since then RTDs have been devised and marketed for the diagnosis of all major TTIs, including HbSAg and HCVAb, and a review on their utilization for transfusion safety in Africa has recently been published [50]. RDTs comply with all the "ASSURED" criteria, and therefore the term RDT is commonly used to describe the POCT devices for infectious diseases diagnosis.

RDTs are essentially based on three main principles: immunochromatography, immunofiltration or immunoconcentration and agglutination. Among these the immunochromatographic strips lateral flow or dipstick - are the most utilized, because of their simplicity and the possibility of single step procedure. The average cost of these tests is today down to a dollar or less. Additionally many of them can utilize capillary blood in place of serum/plasma and can be stored at room temperature. One of the main limitations of RDTs is that they are operator-dependant in several aspects: preparation, interpretation and recording of results. This can explain some discrepancies in reported test accuracy and represents an additional major shortcoming in high-throughput laboratories. Sensitivity of RDTs in TTIs screening is a major concern and this is obviously a crucial aspect in blood safety. As far as analytical sensitivity of HIV RDTs is concerned, as it is in the case of testing series of progressively diluted specimens, the limits of RDTs performance are evident when compared to reference immunoenzymatic tests [51,52]. When clinical sensitivity is considered however, i.e. observations on undiluted samples from patients or from blood donors, many HIV RDTs offer an acceptable level of detection of truly positive samples [45,48]. WHO sets precise standards for acceptability of HIV RDTs: sensitivity $>99 \%$, specificity $>98 \%$ associated to inter-observer variability and invalidity rate both $<5 \%$. A list of commercial kits that comply with these standards is available and regularly updated [53]. Recently a "combined" HIV P24 antigen-antibodies RDT has been marketed with the objective of increasing sensitivity during the early stages of infection. The first independent observations however show a limited diagnostic value of this combo test in the seroconversion period [54].

As far as RDTs for HbSAg and HCVAb are concerned the reported clinical sensitivity is on-average lower than HIV RDTs [45,47,55-59]. The reason for this may be the higher proportion of relatively low-titer reactive samples and the longer window period in the early phases of infection. However also for HbSAg and HCVAb there are kits that show promising results in terms of diagnostic accuracy [60-65] and a
HbSAg RDT has been recently CE marked as it achieves the requested sensitivity of at least $0.125 \mathrm{I} . \mathrm{U} / \mathrm{mL}$ [66].

For all these reasons RDTs for the three major viral TTIs are considered an acceptable alternative for the screening of blood donations where immunoenzymatic tests are not available, i.e. in small peripheral laboratories and in emergency situations [53].

As far as syphilis is concerned Treponemal immunochromatographic RDTs are also available and can replace the more traditional agglutination non-treponemal essays.

In recent years RDTs for Malaria have gained a central role for the diagnosis of this disease, both in high and low-income countries. As blood screening tools RDTs may however fail to detect low grade parasitaemia in asymptomatic "semi-immune" adults, as "healthy" blood donors in malaria endemic countries may often be $[67,68]$.

In general as far as the accuracy of RDTs in field conditions is concerned, it should be remembered that inappropriate storage conditions may heavily affect their performance and this can be a major problem in a tropical environment [69].

In high income countries multiplex nucleic acid amplification testing - NAAT- for HIV, HBV and HCV - is a mandatory additional screening tool. NAAT is able to reduce the infectious window period and therefore also the residual transmission of infectious diseases through blood transfusion. Technologies in molecular diagnostics have rapidly evolved in recent years but very few present operating characteristics that make them appropriate for utilization in peripheral locations of low-income countries. There are however promising NAAT platforms that simplify molecular testing by fully integrating and automating the three processes (sample preparation, amplification, and detection) required for real-time PCR-based molecular testing in a single cartridge containing all necessary elements for the reaction [70]. These instruments are simple enough to be performed reliably even by individuals without a background in nucleic acid diagnostics. Unfortunately no available POC NAAT platform yet includes, among the many testing options, kits for the combined screening of the three main blood-borne viruses. There are however promising and innovative NAAT technologies, such as the loop-mediated isothermal amplification assay (LAMP), with the potential to be sensitive, rapid and user-friendly enough to be utilized as a blood screening tool in remote settings [71].

\section{Pre-transfusion testing}

Pre-transfusion tests, i.e. blood grouping and cross-match, are specific activities of blood bank laboratories. Traditional, simple and cheap ABO-Rh typing, utilizing commercial antisera, can be easily done at the bedside by trained health workers. Dry-reagent typing cards have been marketed for decades and offer an even more simplified typing procedure suitable for bedside blood group confirmation by ward staff $[72,73]$. Innovative paper based blood typing kits are also in the pipeline [74].

Together with $\mathrm{ABO}-\mathrm{Rh}$ typing, however, $\mathrm{WHO}$ recommends the presence of at least the antiglobulin phase compatibility for a complete pre-transfusion testing [75]. Traditional antiglobulin crossmatch, in its tube version, is a relatively simple and affordable test. It requires essential laboratory equipment (low speed centrifuge, clean pipettes, water bath incubator, low power microscope, refrigerator) and reagents (sterile saline solution, antiglobulin serum). The procedure includes several critical steps and requires specific skills in 
Page 4 of 6

interpretation and in problem solving, especially in case of initially reactive results. According to most observations the antiglobulin test is hardly ever encountered in rural hospitals practice in low-income countries, and compatibility testing is generally limited to AB0-Rh typing, often accompanied by room temperature "major" cross-match $[49,76]$.

Since the discovery of antiglobulin testing in the mid $20^{\text {th }}$ century, a major "breakthrough" in immunohematology has been the introduction of gel microcolumn essays which allow a simplified indirect antiglobulin test, with fewer steps, and a more reliable and stable reading of results., The method however still requires specific laboratory skills, equipment and reagents and is therefore not compliant with POCT essential features.

\section{Discussion}

As described there are several point-of-care laboratory technologies that are useful for a safe transfusion practice and appropriate in resource-limited settings. However out of these only serological "rapid diagnostic tests" and some simple hemoglobinometers are available in the field. As described no point-of-care antiglobulin phase pretransfusion tests or equivalent exist or are in the pipeline. Finally, notwithstanding the availability of innovative and simplified NAAT technologies, no POCT kit is yet available in the field of molecular testing of TTIs.

Several factors and barriers that affect the adoption and scaling up of POCT have been identified. These include economic, regulatory, policy-related factors, as well as user/provider perceptions and cultural barriers $[4,77]$.

Recent experiences in the field of POC molecular diagnostics proved however that high-tech technologies can be disseminated globally thanks to a winning combination of elements: a public-private partnership, affordable and "negotiated" prices, a global supply and assistance network, and, not least, a global validation and performance monitoring program $[70,78]$. In other words a strong public health commitment and the contribution of non-profit foundations and international organizations are essential for a successful introduction of new health technologies on a global scale.

If the advantages of POCT in resource-limited settings are selfevident their limitations are also straightforward and must be carefully taken into account when these devices are introduced into clinical practice. Some of the potential disadvantages of POCT are the lack of standardization in obtaining blood samples, the need for additional training and supervision in order to minimize operator subjectivity and the lack of adoption of internal/external quality assessment tools. For these reasons as far as possible rigorous quality assurance procedures, including EQA - External Quality Assessment - should be applied, both for the prevention of procedural and clerical errors as well as for tests validation [79,80]. Moreover newly introduced POC tests and RDTs need to be locally evaluated before supply is established [81]. This is particularly relevant in the field of TTI testing since the quality of local samples, the genotypes and subtypes of circulating infectious agents and other factors may influence the performance of the essay

The problem of poor-quality and counterfeit diagnostics is also an emerging problem in resources-limited settings and health authorities should strengthen their regulatory oversight to the POC tests that are frequently distributed in developing counties [82].

\section{Conclusion}

Blood Transfusions, even if limited to whole blood, are relatively sophisticated and high risk practices in under-resourced environments. The laboratory armamentarium currently available in the blood banks of high-income countries is in many ways impracticable and unaffordable in the majority of rural and remote hospitals in low-income countries.

Existing rapid tests for TTIs screening and portable hemoglobimomethers are POC tests that today contribute to transfusion safety in contexts where more complex and costly devices are unavailable; however more simple, accurate and affordable systems are needed in order to achieve higher standards in all aspects of blood safety in these settings.

Several prototypes are in the pipeline especially in the field of microfluidics and nanotechnologies [83], and in future some of these may prove useful in the field of transfusion medicine. However new technologies for global health cannot be effectively developed without the involvement of the end-users in low-income countries and an active bottom-up approach [84]. Whether these innovations will actually be disseminated and effectively utilized in resource-limited settings will probably depend not only on market dynamics but also on public health policies and funding.

\section{References}

1. Jani IV, Peter TF (2013) How point-of-care testing could drive innovation in global health. N Engl J Med 368: 2319-2324.

2. Lehe JD, Sitoe NE, Tobaiwa O, Loquiha O, Quevedo JI, et al. (2012) Evaluating operational specifications of point-of-care diagnostic tests: a standardized scorecard. PLoS One 7: e47459.

3. Peeling RW, Mabey D (2010) Point-of-care tests for diagnosing infections in the developing world. Clin Microbiol Infect 16: 1062-1069.

4. Pai NP, Vadnais C, Denkinger C, Engel N, Pai M (2012) Point-of-care testing for infectious diseases: diversity, complexity, and barriers in lowand middle-income countries. PLoS Med 9: e1001306.

5. Pai NP, Pai M (2012) Point-of-care diagnostics for HIV and tuberculosis: landscape, pipeline, and unmet needs. Discov Med 13: 35-45.

6. Bates I, McKew S, Sarkinfada F (2007) Anaemia: a useful indicator of neglected disease burden and control. PLoS Med 4: e231.

7. Briggs C, Kimber S, Green L (2012) Where are we at with point-of-care testing in haematology? Br J Haematol 158: 679-690.

8. Stone JE, Simmons WK, Jutsum PJ, Gurney JM (1984) An evaluation of methods of screening for anaemia. Bull World Health Organ 62: 115-120.

9. Aldridge C, Foster HM, Albonico M, Ame SM, Montresor A (2012) Evaluation of the diagnostic accuracy of the Haemoglobin Colour Scale to detect anaemia in young children attending primary healthcare clinics in Zanzibar. Trop Med Int Health 17: 423-429.

10. Barduagni P, Ahmed AS, Curtale F, Raafat M, Soliman L (2003) Performance of Sahli and colour scale methods in diagnosing anaemia among school children in low prevalence areas. Trop Med Int Health 8: 615-618.

11. Tyburski EA, Gillespie SE, Stoy WA, Mannino RG, Weiss AJ, et al. (2014) Disposable platform provides visual and color-based point-of-care anemia self-testing. J Clin Invest 124: 4387-4394.

12. Desai MR, Phillips-Howard PA, Terlouw DJ, Wannemuehler KA, Odhacha A, et al. (2002) Recognition of pallor associated with severe anaemia by primary caregivers in western Kenya. Tropical Medicine and International Health 7: 831-839.

13. von Schenck H, Falkensson M, Lundberg B (1986) Evaluation of "HemoCue," a new device for determining hemoglobin. Clin Chem 32: 526-529. 
14. Lardi AM, Hirst C, Mortimer AJ, McCollum CN (1998) Evaluation of the HemoCue for measuring intra-operative haemoglobin concentrations: a comparison with the Coulter Max-M. Anaesthesia 53: 349-352.

15. Nkrumah B, Nguah SB, Sarpong N, Dekker D, Idriss A, et al. (2011) Hemoglobin estimation by the HemoCue ${ }^{\infty}$ portable hemoglobin photometer in a resource poor setting. BMC Clin Pathol 11: 5.

16. Goldman M, Uzicanin S, Yi QL, Acker J, Ramirez-Arcos S (2012) Validation and implementation of a new hemoglobinometer for donor screening at Canadian Blood Services. Transfusion 52: 1607-1613.

17. Medina Lara A, Mundy C, Kandulu J, Chisuwo L, Bates I (2005) Evaluation and costs of different haemoglobin methods for use in district hospitals in Malawi. J Clin Pathol 58: 56-60.

18. Critchley J, Bates I (2005) Haemoglobin colour scale for anaemia diagnosis where there is no laboratory: A systematic review. Int J Epidemiol 34: 1425-1434.

19. Montresor A, Ramsan M, Khalfan N, Albonico M, Stoltzfus RJ, et al. (2003) Performance of the Haemoglobin Colour Scale in diagnosing severe and very severe anaemia. Trop Med Int Health 8: 619-624.

20. van Lerberghe W, Keegels G, Cornelis G, Ancona C, Mangelschots E, et al. (1983) Haemoglobin measurement: the reliability of some simple techniques for use in a primary health care setting. Bull World Health Organ 61: 957-965.

21. Mustaring, Tranggana S, Daud D (1990) Capillary microhematocrit measurement as a screening test for anemia in children. Paediatr Indones 30: 241-247.

22. Young PC, Hamill B, Wasserman RC, Dickerman JD (1986) Evaluation of the capillary microhematocrit as a screening test for anemia in pediatric office practice. Pediatrics 78: 206-209.

23. Srivastava A, Koul V, Dwivedi SN, Upadhyaya AD, Ahuja A, et al . (2014) Performance analysis of newly developed point-of-care hemoglobinometer (TrueHb) against an automated hematology analyzer (Sysmex XT 1800i) in terms of precision in hemoglobin measurement. Int J Lab Hematol.

24. Gayat E, Bodin A, Sportiello C, Boisson M, Dreyfus JF, et al. (2011) Performance evaluation of a noninvasive hemoglobin monitoring device. Ann Emerg Med 57: 330-333.

25. Knutson T, Della-Giustina D, Tomich E, Wills B, Luerssen E, et al. (2013) Evaluation of a new nonnvasive device in determining hemoglobin levels in emergency department patients. West J Emerg Med 14: 283-286.

26. Rice MJ, Gravenstein N, Morey TE (2013) Noninvasive hemoglobin monitoring: how accurate is enough? Anesth Analg 117: 902-907.

27. Erhabor O, Richardson G, Mohammed I, Thornton C, Bark J, et al. (2013) Evaluation of the QBC Star centrifugal three-part differential haematology system. Br J Biomed Sci 70: 67-74.

28. Singh A, Dubey A, Sonker A, Chaudhary R (2015) Evaluation of various methods of point-of-care testing of haemoglobin concentration in blood donors. Blood Transfus 13: 233-239.

29. Sawant RB, Bharucha ZS, Rajadhyaksha SB (2007) Evaluation of hemoglobin of blood donors deferred by the copper sulphate method for hemoglobin estimation. Transfus Apher Sci 36: 143-148.

30. Punguyire D, Iserson KV, Stolz U, Apanga S (2013) Bedside whole-blood clotting times: validity after snakebites. J Emerg Med 44: 663-667.

31. Sano-Martins IS, Fan HW, Castro SC, Tomy SC, Franca FO, et al. (1994) Reliability of the simple 20 minute whole blood clotting test (WBCT20) as an indicator of low plasma fibrinogen concentration in patients envenomed by Bothrops snakes. Toxicon 32: 1045-1050.

32. Isbister GK, Maduwage K, Shahmy S, Mohamed F, Abeysinghe C, et al. (2013) Diagnostic 20-min whole blood clotting test in Russell's viper envenoming delays antivenom administration. QJM 106: 925-932.

33. Wieloch M, Hillarp A, Strandberg K, Nilsson C, Svensson PJ (2009) Comparison and evaluation of a Point-of-care device (CoaguChek XS) to Owren-type prothrombin time assay for monitoring of oral anticoagulant therapy with warfarin. Thromb Res 124: 344-348.

34. David JS, Levrat A, Inaba K, Macabeo C, Rugeri L, et al. (2012) Utility of a point-of-care device for rapid determination of prothrombin time in trauma patients: a preliminary study. J Trauma Acute Care Surg 72: 703-707.

35. Goodnough LT, Hill CC (2012) Use of point-of-care testing for plasma therapy. Transfusion 52 Suppl 1: 56S-64S.

36. Enriquez LJ, Shore-Lesserson L (2009) Point-of-care coagulation testing and transfusion algorithms. Br J Anaesth 103 Suppl 1: i14-22.

37. Meybohm P, Zacharowski K, Weber CF (2013) Point-of-care coagulation management in intensive care medicine. Crit Care 17: 218.

38. Whiting D, DiNardo JA (2014) TEG and ROTEM: technology and clinical applications. Am J Hematol 89: 228-232.

39. Solomon C, Collis RE, Collins PW (2012) Haemostatic monitoring during postpartum haemorrhage and implications for management. $\mathrm{Br} \mathrm{J}$ Anaesth 109: 851-863.

40. Brazzel C (2013) Thromboelastography-guided transfusion Therapy in the trauma patient. AANA J 81: 127-132.

41. Mallett SV, Armstrong M (2015) Point-of-care monitoring of haemostasis. Anaesthesia 70 Suppl 1: 73-77, e25-6.

42. Ala F, Allain JP, Bates I, Boukef K, Boulton F, et al. (2012) External financial aid to blood transfusion services in sub-Saharan Africa: a need for reflection. PLoS Med 9: e1001309.

43. Spinella PC (2008) Warm fresh whole blood transfusion for severe hemorrhage: U.S. military and potential civilian applications. Crit Care Med 36: S340-345.

44. http://www.ncbi.nlm.nih.gov/books/NBK142990.

45. Mbanya D (2013) Use of quality rapid diagnostic testing for safe blood transfusion in resource-limited settings. Clin Microbiol Infect 19: 416-421.

46. La Raja M (2009) Rapid tests for blood donor screening in Africa. Transfusion 49: 2773-2774.

47. Allain JP, Lee H (2005) Rapid tests for detection of viral markers in blood transfusion. Expert Rev Mol Diagn 5: 31-41.

48. Owusu-Ofori S, Temple J, Sarkodie F, Anokwa M, Candotti D, et al. (2005) Predonation screening of blood donors with rapid tests: implementation and efficacy of a novel approach to blood safety in resource-poor settings. Transfusion 45: 133-140.

49. Bugge HF, Karlsen NC, Oydna E, Rake MM, Wexels N, et al. (2013) A study of blood transfusion services at a district hospital in Malawi. Vox Sang 104: 37-45.

50. Pruett CR, Vermeulen M, Zacharias P, Ingram C, Tayou Tagny C, et al. (2015) The use of rapid diagnostic tests for transfusion infectious screening in Africa: a literature review. Transfus Med Rev 29: 35-44.

51. Laperche S, Boukatou G, Kouegnigan L, Nébié Y, Boulahi MO, et al. (2009) Transfusion safety on the African continent: an international quality control of virus testing in blood banks. Transfusion 49: 1600-1608.

52. Bloch EM, Shah A, Kaidarova Z, Laperche S, Lefrere JJ, et al. (2014) A pilot external quality assurance study of transfusion screening for HIV, HCV and HBsAG in 12 African countries. Vox Sang 107: 333-342.

53. http://www.who.int/diagnostics_laboratory/procurement/ 120524_v7_product_eligible_for_who_procurement_2012.pdf.

54. Laperche S, Leballais L, Ly TD, Plantier JC (2012) Failures in the detection of HIV p24 antigen with the Determine HIV-1/2 Ag/Ab Combo rapid test. J Infect Dis 206: 1946-1947.

55. Shivkumar S, Peeling R, Jafari Y, Joseph L, Pant Pai N (2012) Accuracy of rapid and point-of-care screening tests for hepatitis $\mathrm{C}$ : a systematic review and meta-analysis. Ann Intern Med 157: 558-566.

56. Firdaus R, Saha K, Sadhukhan PC (2013) Rapid immunoassay alone is insufficient for the detection of hepatitis $\mathrm{C}$ virus infection among highrisk population. J Viral Hepat 20: 290-293.

57. Scheiblauer H, El-Nageh M, Diaz S, Nick S, Zeichhardt H, et al. (2010) Performance evaluation of 70 hepatitis $B$ virus (HBV) surface antigen (HBsAg) assays from around the world by a geographically diverse panel with an array of HBV genotypes and HBsAg subtypes. Vox Sang 98: 403-414. 
Citation: Raja ML, Musi R, Fattorini M, Piva E, Putoto G (2015) Point of Care Testing and Transfusion Safety in Resource Limited Settings: A Review. J Blood Disorders Transf 6: 269. doi:10.4172/2155-9864.1000269

Page 6 of 6

58. Khuroo MS, Khuroo NS, Khuroo MS (2014) Accuracy of Rapid Point-ofCare Diagnostic Tests for Hepatitis B Surface Antigen-A Systematic Review and Meta-analysis. J Clin Exp Hepatol 4: 226-240.

59. Tagny CT, Mbanya D, Murphy EL, Lefrère JJ, Laperche S (2014) Screening for hepatitis $\mathrm{C}$ virus infection in a high prevalence country by an antigen/antibody combination assay versus a rapid test. J Virol Methods 199: 119-123.

60. Cha YJ, Park Q, Kang ES, Yoo BC, Park KU, et al. (2013) Performance evaluation of the OraQuick hepatitis $\mathrm{C}$ virus rapid antibody test. Ann Lab Med 33: 184-189.

61. Kant J, Möller B, Heyne R, Herber A, Böhm S, et al. (2013) Evaluation of a rapid on-site anti-HCV test as a screening tool for hepatitis $\mathrm{C}$ virus infection. Eur J Gastroenterol Hepatol 25: 416-420.

62. O'Connell RJ, Gates RG, Bautista CT, Imbach M, Eggleston JC, et al. (2013) Laboratory evaluation of rapid test kits to detect hepatitis C antibody for use in predonation screening in emergency settings. Transfusion 53: 505-517.

63. Franzeck FC, Ngwale R, Msongole B, Hamisi M, Abdul O, et al. (2013) Viral hepatitis and rapid diagnostic test based screening for HBsAg in HIV-infected patients in rural Tanzania. PLoS One 8: e58468.

64. Davies J, van Oosterhout JJ, Nyirenda M, Bowden J, Moore E, et al. (2010) Reliability of rapid testing for hepatitis B in a region of high HIV endemicity. Trans R Soc Trop Med Hyg 104: 162-164.

65. Kosack CS, Nick S, Shanks L (2014) Diagnostic accuracy evaluation of the ImmunoFlow HCV rapid immunochromatographic test for the detection of hepatitis C antibodies. J Virol Methods 204: 6-10.

66. Chevaliez S, Challine D, Naija H, Luu TC, Laperche S, et al. (2014) Performance of a new rapid test for the detection of hepatitis B surface antigen in various patient populations. J Clin Virol 59: 89-93.

67. Falade CO, Nash O, Akingbola TS, Michael OS, Olojede F, et al. (2009) Blood banking in a malaria-endemic area: evaluating the problem posed by malarial parasitaemias. Ann Trop Med Parasitol 103: 383-392.

68. Owusu-Ofori AK, Betson M, Parry CM, Stothard JR, Bates I (2013) Transfusion-transmitted malaria in Ghana. Clin Infect Dis 56: 1735-1741.

69. Bienek DR, Charlton DG (2012) The effect of simulated field storage conditions on the accuracy of rapid user-friendly blood pathogen detection kits. Mil Med 177: 583-588.

70. Lawn SD, Mwaba P, Bates M, Piatek A, Alexander H, et al. (2013) Advances in tuberculosis diagnostics: the Xpert MTB/RIF assay and future prospects for a point-of-care test. Lancet Infect Dis 13: 349-361.
71. Nyan DC, Ulitzky LE, Cehan N, Williamson P, Winkelman V, et al. (2014) Rapid detection of hepatitis B virus in blood plasma by a specific and sensitive loop-mediated isothermal amplification assay. Clin Infect Dis 59: 16-23.

72. ELDON K (1956) Experience with $\mathrm{ABO}$ and Rh blood-grouping cards (Eldon cards). Br Med J 2: 1218-1220.

73. Giebel F, Picker SM, Gathof BS (2008) Evaluation of Four Bedside Test Systems for Card Performance, Handling and Safety. Transfus Med Hemother 35: 33-36.

74. Noiphung J, Talalak K, Hongwarittorrn I, Pupinyo N, Thirabowonkitphithan P, et al. (2015) A novel paper-based assay for the simultaneous determination of Rh typing and forward and reverse $\mathrm{ABO}$ blood groups. Biosens Bioelectron 67: 485-489.

75. http://apps.who.int/iris/bitstream/10665/38905/1/9241544457_eng.pdf.

76. Lund TC, Hume H, Allain JP, McCullough J, Dzik W (2013) The blood supply in Sub-Saharan Africa: needs, challenges, and solutions. Transfus Apher Sci 49: 416-421.

77. Mabey D, Peeling RW, Ustianowski A, Perkins MD (2004) Diagnostics for the developing world. Nat Rev Microbiol 2: 231-240.

78. [No authors listed] (2007) Neglected diagnostics. Nat Methods 4: 877-878.

79. Parekh BS, Kalou MB, Alemnji G, Ou CY, Gershy-Damet GM, et al. (2010) Scaling up HIV rapid testing in developing countries: comprehensive approach for implementing quality assurance. Am J Clin Pathol 134: 573-584.

80. Plebani M, Lippi G (2014) Point of care testing: evolving scenarios and innovative perspectives. Clin Chem Lab Med 52: 309-311.

81. Drain PK, Hyle EP, Noubary F, Freedberg KA, Wilson D, et al. (2014) Diagnostic point-of-care tests in resource-limited settings. Lancet Infect Dis 14: 239-249.

82. Mori M, Ravinetto R, Jacobs J (2011) Quality of medical devices and in vitro diagnostics in resource-limited settings. Trop Med Int Health 16: 1439-1449.

83. Yager P, Domingo GJ, Gerdes J (2008) Point-of-care diagnostics for global health. Annu Rev Biomed Eng 10: 107-144.

84. Howitt P, Darzi A, Yang GZ, Ashrafian H, Atun R, et al. (2012) Technologies for global health. Lancet 380: 507-535. 